\title{
Crecimiento económico, concentración del ingreso y reducción de la pobreza: Evidencia en Ecuador de la Hipótesis de Bourguignon
}

\section{Economic growth, income concentration and poverty reduction: Evidence in Ecuador of the Bourguignon hypothesis}

\author{
Ramiro Efraín Villarruel Meythaler ${ }^{1}$, David Hernán Echeverría Villafuerte ${ }^{1}$, Mónica Andrea Bedoya Ramos ${ }^{1}$, \\ Evelyn Sofía Moreta Saraguro ${ }^{1}$ \\ ${ }^{1}$ Universidad Central del Ecuador, Ecuador \\ *revillarruel@uce.edu.ec
}

DOI: https://doi.org/10.26871/killkanasocial.v4i3.624

\begin{abstract}
Resumen
En la presente investigación se analizan los efectos que tiene el crecimiento económico, medido con el ingreso per cápita familiar de los hogares, y la desigualdad por ingresos, medida con el coeficiente de Gini, en la incidencia de la pobreza en Ecuador. Durante el periodo de análisis 2007-2017, en uso de los datos de la Encuesta Nacional de Empleo, Desempleo y Subempleo -ENEMDU-, se ha demostrado que las variaciones del crecimiento económico y de la desigualdad de ingresos si influyen significativamente en la incidencia de la pobreza. Mediante el cálculo de las elasticidades, provisto por la estimación de los modelos econométricos de regresión logarítmica múltiple a nivel nacional y provincial, se ha determinado que el coeficiente de Gini resulta ser más elástico que el ingreso per cápita de los hogares frente a la incidencia de la pobreza en el país y en la mayoría de las provincias. Acorde a la Hipótesis de Bourguignon (2004), esto implica que, las políticas distributivas y redistributivas en el país son más eficaces para culminar con la pobreza debido a los niveles de desigualdad que posee Ecuador. No obstante, tal situación no significa que se deba obviar las políticas de crecimiento económico, las mismas que también son necesarias para la generación de recursos que permitan la consecución de los objetivos nacionales de desarrollo.
\end{abstract}

Palabras clave: pobreza, crecimiento económico, desigualdad, ingreso, modelo de regresión.

\begin{abstract}
The present investigation analyzes the effects of economic growth, measured with household per capita income, and income inequality, measured with Gini coefficient, on the incidence of poverty in Ecuador. During the period 2007-2017, using data from the Encuesta Nacional de Empleo, Desempleo y Subempleo-ENEMDU-, it was shown that variations in economic growth and income inequality do have a significant impact on poverty. By calculating the elasticities, provided by the estimation of a multiple logarithmic regression model at the national and provincial levels, it was determined that the Gini coefficient turns out to be more elastic than the per capita income of households compared to poverty in the country and most of the provinces. According to the Bourguignon Hypothesis (2004), this implies that the distributive and redistributive policies in the country are more effective in ending poverty due to the levels of inequality that Ecuador has. However, such a situation does not mean that economic growth policies should be ignored, which are also necessary for the generation of resources that allow the achievement of national objectives.
\end{abstract}

Keywords: poverty, economic growth, inequality, income, logarithmic regression model.

\section{Introducción}

La pobreza es el problema más importante por resolver para la humanidad. El primer Objetivo de Desarrollo Sustentable propone "poner fin a la pobreza en todas sus formas" hasta el año 2030 -Naciones Unidas- (2015). Este problema impide que las personas puedan alcanzar a satisfacer necesidades básicas como el acceso al agua potable y saneamiento, educación, salud, alimentación, entre otras. Los factores que influyen en la imposibilidad de supe- rar este problema son: Modelo comercial multinacional, corrupción, cambio climático, enfermedades y epidemias, desigualdad en el reparto de recursos, crecimiento de la población, conflictos armados, discriminación de género, desperdicio de alimentos, fatal de agilidad en las acciones para erradicar la pobreza por parte de los países desarrollados -Oxfam- (2019). Se puede colegir que la pobreza y los factores asociados enmarcan un contexto complejo para los países en vías de desarrollo, los Estados no cuentan con los 
recursos suficientes para invertir en infraestructura, servicios y bienes públicos calidad que mejoren sustancialmente la calidad de vida de la población en condición de pobreza y pobreza extrema.

Según el Programa de las Naciones Unidas para el Desarrollo -PNUD- (2019), las personas que se encuentran en situación de pobreza extrema disminuyeron en más de la mitad dentro del periodo 1990-2015. Actualmente, 789 millones de personas viven por debajo del umbral de pobreza internacional, 1,90 dólares diarios -Naciones Unidas(2015), mientras que, 1.300 millones viven en pobreza multidimensional y el $50 \%$ son menores de edad -PNUD(2019). Lamentablemente trabajar tampoco impide superar la pobreza, en 2018 se estimó que el $8 \%$ de los trabajadores a nivel mundial viven en situación de extrema pobreza. En el aspecto regional la Comisión Económica para América Latina y el Caribe -CEPAL- (2017), determina que dentro del periodo 2002-2014 la pobreza disminuyó en un valor considerable, pero el ritmo de decrecimiento al pasar los años fue menor. La pobreza y la pobreza extrema en 2015 y 2016 aumentaron, en este último año las personas en esta situación llegaron a 186 millones, 30,7\% de la población total.

En el Plan Nacional de Desarrollo de Ecuador 20172021, se establecieron tres metas con respecto a la pobreza y la desigualdad el 2021: Erradicar la incidencia de pobreza extrema por ingresos reduciéndola del 8,7\% al $3,5 \%$; reducir la tasa de pobreza multidimensional desde el $35,1 \%$ al 27,4\%; y, disminuir la relación de ingresos entre el decil más pobre y el decil más rico desde 24,3 a menos de 20 veces (Secretaría Nacional de Planificación y Desarrollo, Senplades, 2017). En Ecuador, la población en situación de pobreza según el Instituto Nacional de Estadística y Censos -INEC- (s.f.), para diciembre de 2017 se ubicó en $21,5 \%$ del total de la población, mientras que la pobreza extrema se ubicó en 7,9\% y el coeficiente de Gini alcanzó 0,4594. En el marco de lo expuesto, la pobreza debe constituir un eje central de la política pública, para lo cual, se puede tener en cuenta dos tipos de políticas que estén encaminadas a su disminución: Políticas de crecimiento, políticas de distribución o disminución de la desigualdad, o ambas de manera simultáneas. Bourguignon (2004) plantea la existencia del triángulo pobreza, el crecimiento y desigualdad; y, expone que la mejor forma de incidir en la disminución de la pobreza es a través de la combinación de las dos políticas. Las políticas de crecimiento promueven la obtención de recursos que, mediante las políticas de distribución, los reparten de la mejor manera. Pese a esto, una de las dos políticas resulta más eficaz dependiendo del entorno. Este estudio mostrará evidencia al respecto para el caso de Ecuador durante el periodo 2007-2017.

El artículo se compone de apartados. Se realiza una revisión bibliográfica sobre la Hipótesis de Bourguignon: la relación triangular entre crecimiento económico, la desigualdad y la pobreza, donde a su vez, se exponen datos sobre la situación de las tres variables de estudio en Ecuador durante el periodo de estudio. Posteriormente se presenta los resultados del modelo econométrico de regresión logarítmica múltiple y el cálculo de elasticidades nacional y provincial, pobreza-ingreso y pobreza-desigualdad. Por último, a la luz de los resultados, se aborda brevemente los retos políticos, conclusiones y recomendaciones encaminadas a la erradicación a la pobreza.

\section{Marco teórico o antecedentes}

Teóricamente esta investigación se basa en la Hipótesis de Bourguignon, que explica la relación triangular existente entre crecimiento económico, la desigualdad y la pobreza, además de otras investigaciones en torno a los tres ejes que apoyan la hipótesis. Para el estudio empírico se usan los datos de la Encuesta Nacional de Empleo, Desempleo y Subempleo -ENEMDU- a nivel nacional y provincial, para crear una base de datos de panel para el periodo 2007-2017, que permite estimar las elasticidades de las variables explicativas frente a la pobreza, mediante un modelo econométrico logarítmico múltiple. La finalidad es contrastar la teoría expuesta con resultados obtenidos para el caso de Ecuador.

\subsection{Hipótesis de Bourguignon}

Existe un amplio debate sobre el efecto que ejercen el crecimiento económico y la desigualdad sobre la pobreza. Parece haber mayor consenso entre quienes apoyan que el crecimiento económico es indispensable para la reducción de la pobreza medida por los ingresos. No obstante, en países donde la concentración de los ingresos es muy alta, se requiere de medidas distributivas que permitan hacer un uso eficaz del aumento de ingresos. Bourguignon (2004) expone que ambos ejes, crecimiento y distribución, son necesarios para la merma del problema de la pobreza y que la verdadera cuestión es saber en qué medida aplicar políticas entorno a ello a manera de estrategia de desarrollo.

Bourguignon (2004) determina una función de distribución del ingreso, asumiendo una distribución logarítmica normal en la que las variaciones de la pobreza se deben a dos efectos, el del crecimiento y distribución. Ambos se ven reflejados en la Figura 1 donde, se declaran pobres a las personas que perciben menos de un dólar diario y que están por debajo de la función de distribución. En un principio, únicamente por el de efecto del crecimiento en el que los ingresos medios de toda la población, la curva de la función de distribución inicial se traslada horizontalmente hacia la derecha sobre el eje de ingresos hasta la curva intermedia o (I). Posteriormente, con el efecto distributivo, el cual produce cambios en el ingreso relativo y, el ingreso promedio permanece invariable, la curva se traslada a la de la distribución final, provocando en una disminución significativa de la pobreza. 


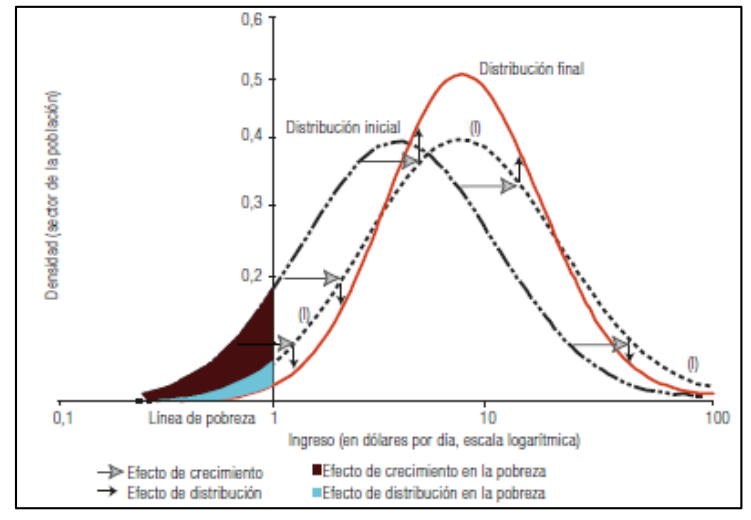

Figura 1: Hipótesis de Bourguignon Fuente: Bourguignon, 2004

Así Bourguignon concluye que la simultaneidad en el uso de políticas de crecimiento y distribución es muy importante si se quiere disminuir la pobreza en términos absolutos, sin embargo, la medida y temporalidad en la que se apliquen dependerá de la zona en donde se quiera ejecutar tales políticas como parte de una estrategia de desarrollo.

\subsection{Crecimiento económico y su relación con la pobreza}

La idea de apoyar las políticas de crecimiento económico es aceptada cuando, como eje estratégico, está disminuir la pobreza, ya sea mermando el número absoluto de pobres o que el ingreso de los pobres crezca más rápido que del resto de la población, éste último generalmente viene acompañado de enfoques distributivos. Así, Deininger \& Squire (1996) en un estudio realizado a 95 países alrededor del mundo determinaron que existe una fuerte relación entre el crecimiento económico y la reducción de la pobreza, dado que 91 países con crecimiento económico promedio, en un periodo de diez años, presentaron incrementos en el ingreso del quintil más pobre de la población.

Posteriormente, Ravallion \& Chen (1997) llevaron a cabo estudios en 45 países sobre elasticidades del crecimiento económico frente a la pobreza, y encontraron que efectivamente existe una relación robusta entre tales variables y rechazan la idea de que el crecimiento de los niveles promedio de vida de la población incrementen la desigualdad. Sin embargo, notaron que la elasticidad pasaba de $0.6 \%$ en países con altos niveles de concentración del ingreso, al $4.3 \%$ en países con niveles bajos de desigualdad; es decir, el crecimiento económico es más eficaz para reducir la pobreza cuando el ingreso está menos concentrado, por ejemplo, países que cuenten con políticas distributivas que reduzcan esa concentración.

En esa línea, Stewart (2000) reflejó como gracias a un enfoque de desigualdad considerado por cada gobierno, el incremento de la riqueza afectaba distintamente a la pobreza. Stewart realizó investigaciones sobre el efecto porcentual de la tasa de crecimiento en Malasia y Zambia. En el primer caso, la pobreza disminuyó en 3.4\%, país que actualmente presenta un Índice de Desarrollo Humano
-IDH- del 0.82, mientras que en el segundo solo mermó el $0.21 \%$ con un IDH del 0.57. También, Chen \& Wang (2001), en una investigación elaborada en China, examinan nuevamente ambas variables y determinan que en el periodo de 1900-1999 el crecimiento económico disminuyó notoriamente la pobreza, pero que, a excepción de un periodo, la desigualdad aumentó debido a la concentración del ingreso en el grupo de mayores ingresos.

A nivel de Latinoamérica también se ha estudiado los efectos del crecimiento económico sobre la pobreza. Expósito, Fernández \& Velasco (2017), en un estudio sobre la evaluación de los sistemas económicos frente a la reducción de la pobreza y la desigualdad durante el periodo 20002014, encuentran que el crecimiento económico no ha sido capaz de reducir eficientemente los niveles de pobreza. Esto gracias a que el enfoque político se ha direccionado en mayor parte a la acumulación de capital, y de paso, de concentración de riqueza, relegando el papel de la política social, el cual ha estado a expensas de ingresos dependientes del mercado internacional lo que, a su vez, refleja deficiencias estructurales productivas.

\subsection{Desigualdad y su relación con la pobreza}

La pobreza y la desigualdad han permanecido a nivel mundial, algunos reconocidos economistas promulgaron en el Banco Mundial -BM- (1990), que la pobreza es un problema social que hay que tomar en cuenta, y más bien la desigualdad es un beneficio para la dinamización del mercado. Pese a ello, la pobreza siempre va de la mano con la desigualdad. Un país desigual no significa un país pobre, pero un país pobre siempre será desigual. En varios estudios se analiza que existe una disminución de la pobreza cuando hay políticas de crecimiento junto con estrategias de distribución del ingreso. Para Bourguignon (2004) estas últimas son más útiles que las del crecimiento para dicha reducción. Es así como se denota la relación que existe entre pobreza y desigualdad.

Cuando se habla de la desigualdad en la distribución también se torna fundamental mencionar los factores asociados, y entre los cuales se puede citar injusticia e ineficiencia en política fiscal, problemas de corrupción, etc. La desigualdad más debatida suele ser la que se deriva de la concentración del ingreso. Para Verdera (2005) esta última afirmación está en discusión, pues necesariamente la pobreza tiene una relación directa con la concentración.

El crecimiento no es la única forma de reducción de la pobreza. Stiglitz (2015) menciona que el mercado es endógeno de la política y, por lo general, la política favorece a los más ricos a costa de los más pobres. Toda sociedad debe estar inmersa en un marco jurídico e instituciones que establezcan normativas para que la misma funcione, pero al igual que las políticas, este marco está ajustado a las necesidades de las personas más acaudaladas, lo que trae repercusiones en la distribución del ingreso, recayendo el peso en la pobreza. Estos beneficios causan concentración del ingreso, según un informe del Fondo Monetario Inter- 
nacional -FMI- (2014), declara que el $1 \%$ que cuenta con más ingreso en el mundo, tiene un crecimiento de la riqueza del $0,1 \%$ más rico.

Piketty (2013) hace énfasis en un análisis sobre la concentración de la riqueza y, por tanto, de la desigualdad del mundo causante de pobreza. Un aumento de la desigualdad se da, debido a la mala distribución del capital permitiendo que los patrimonios crezcan desmesuradamente. Dentro de su análisis encuentra también que la tasa de crecimiento económico de un país es menor que la tasa de rendimiento del capital, este es el motivo por el cual la desigualdad se mantiene constante. La razón por la cual la pobreza no cesa es que este ingreso que se acumula en los patrimonios de los más ricos y que es producido por los más pobres, se mantiene constante y sin distribución, es decir, una concentración a expensas de la pobreza.

\subsection{Relación entre crecimiento económico y desigualdad}

Pensar en que el crecimiento económico lograría la disminución de la desigualdad, además de la erradicación de otras problemáticas sociales, es una idea que investigadores han abordado hace décadas. Tal es el caso de Kuznets (1955) quién enfatiza sobre el impacto del crecimiento económico, medido a través del ingreso per cápita, en la desigualdad, medida a través del coeficiente de Gini. López (2017) afirmó que Kuznets comenzó su hipótesis sabiendo que los países en transformación de preindustriales a industriales presentaban al crecimiento económico y a la desigualdad con una tendencia a tener una relación en forma de U invertida. Esta hipótesis se maneja con que los países pobres son igualitarios al comienzo, no obstante, a medida que existe crecimiento se va dando mayor concentración del ingreso y, por lo tanto, mayor desigualdad.

Pese a ello, ante un crecimiento posterior esta concentración se va disolviendo y la desigualdad nivelando para convertirse en un país más igualitario, ya que llegaría un punto en que tal desigualdad caería gracias al traspaso de mano de obra del sector agrícola al sector industrial, mejorando progresivamente sus ingresos, lo que resulta en mayor equidad económica. Lo antes mencionado ha dado paso a observar si esta hipótesis se cumple en la práctica. En los países desarrollados, como en los países en vías de desarrollo, la concentración del ingreso tiene una tendencia igual, es decir que la curva no sigue su curso completo, se queda estancada en el punto alto de la curva, existiendo una contradicción en la hipótesis de Kuznets empíricamente, y al existir mayor desigualdad se ha observado mayor pobreza en la mayoría de los países (López, 2017).

Por lo expuesto, se presenta una verdadera preocupación sobre el cómo se está repartiendo el ingreso generado por el crecimiento económico. La desigualdad tiene mayores posibilidades de permanecer constante o aumentar aún con una mejor posición a nivel macroeconómico. Bruno, Ravallion \& Squire (1996) dan una nueva perspectiva con respecto a la desigualdad, ellos analizaron datos sobre el ingreso per cápita y el coeficiente de Gini de 44 países entre los años sesenta y noventa. En este estudio critican la hipótesis de Kuznets, ya que, no se muestran indicios de la curva invertida. Además, denotan que existen otros determinantes que influyen en estas dos situaciones, como, el entorno en el que se vive y las políticas de distribución en ámbitos como educación, salud y nutrición. Estas últimas hacen que se formen activos productivos que guíen a la salida de la pobreza y se conviertan en instrumentos para lograr que el crecimiento sea mayor sin distorsiones.

En esta línea, Acemoglu, Johson \& Robinson (2005) hablan de que las instituciones políticas y económicas son dependientes, siendo estas últimas las que influencian en la redistribución del ingreso y, por lo tanto, también en los resultados económicos. Por ejemplo, para el año 2012, América Latina mostró un promedio en términos de desigualdad de 0,539 y un PIB per cápita de 10.047 dólares, mientras que en 2015 fue de 0,469 y 8.723 dólares respectivamente. El índice disminuyó $1.2 \%$ anual entre los años 2008 y 2012, en tanto que para el periodo 2012 y 2015 la reducción fue de $0.6 \%$ anual (CEPAL, 2017). Aún, cuando el ingreso disminuyó, su distribución se vio mejorada. Así, las políticas de desigualdad en distribución deben ir de la mano con el crecimiento, tomando en cuenta los factores antes mencionados para que tenga resultados de incidencia dentro de la pobreza.

Por otro lado, la investigación de Kalwij \& Verschoor (2004), con datos de panel de 58 países en desarrollo en el periodo 1980-1998, recalca la importancia del efecto del crecimiento económico para la disminución de la pobreza. Sin embargo, ese efecto varía de acuerdo con la región, como -0.71 para África y -2.27 para Medio Oriente y África del Norte. Además, al incluir el análisis de la desigualdad por ingresos, concluye que el efecto que tiene tal desigualdad es mayor en regiones donde existe amplia población que vive con un ingreso cerca de la línea de pobreza. Por ejemplo, en las regiones de Europa Oriental y Asia Central, una combinación de crecimiento y distribución del ingreso provocan mayor efecto en las variaciones de la pobreza, que solo un enfoque de crecimiento.

En el ámbito regional, Andrade, Marinho \& Lima (2017), en una investigación llevada a cabo en Brasil, con datos de panel de todos los estados brasileños en el periodo 1995-2009, afirman que, a nivel de país las políticas distributivas deben ser priorizadas para la eliminación de la desigualdad, ya que, tendrán un mayor efecto en la reducción de la pobreza. Ello considerando que Brasil es un país con bajo nivel de desarrollo inicial y posee altos niveles de desigualdad, haciendo el trabajo de reducir la pobreza aún más complicado. A nivel de estados, se encuentra que en aquellas donde el ingreso promedio es mayor, las políticas de crecimiento resultarían más efectivas; mientras que, en regiones donde la desigualdad es más alta, las políticas distributivas serían las adecuadas. 


\subsection{Crecimiento económico, desigualdad y pobreza en Ecuador}

El crecimiento económico ecuatoriano será descrito a partir del ingreso per cápita familiar, el cual representa según el INEC (2018) "una medida de bienestar económico que capta el ingreso corriente total que dispone el hogar y es repartido para cada de uno de los miembros de éste" (p.02). Como se muestra en la Tabla 1, a nivel nacional, el ingreso per cápita de los hogares creció en un $61,69 \%$ en diez años, debido a la reestructuración política y económica que atravesó Ecuador desde el 2007 y el boom en los precios de las materias primas. Además, hay que considerar que en este periodo hubo muchos cambios y sucesos en la economía nacional e internacional, por ejemplo, la crisis económica mundial de 2008 y el derrumbe de la burbuja inmobiliaria que provocó la bancarrota en varias instituciones financieras norteamericanas, y la crisis bursátil en la mayor parte de los países desarrollados (Revista Gestión, 2013).

La desigualdad en Ecuador ha sido difícil de disminuir, la misma que está representada por el coeficiente de Gini. Este "Mide el grado de desigualdad de una variable en una distribución. En este caso, la desigualdad está en términos del ingreso per cápita del hogar" (INEC, 2018, p.13). A nivel nacional el coeficiente de Gini ha disminuido considerablemente en un $16,62 \%$ en los diez años del periodo. Sin embargo, hay que considerar que, aunque haya crecimiento económico no necesariamente hay menos desigualdad. En el año 2013 existe un crecimiento del $4,95 \%$ de la economía, mientras que en el mismo año se observa en la Tabla 1 como el coeficiente de Gini aumenta de 0,48 a 0,49 .

La pobreza está medida a través de la línea de pobreza por ingreso, que "Para diciembre 2018, se considera a una persona pobre por ingresos si percibe un ingreso familiar per cápita menor a 84,79 dólares mensuales" (INEC, 2018, p.4). A nivel nacional, la pobreza disminuyó en un $41,59 \%$ en diez años. Los picos más altos fueron en los años 2009 y 2015. El primero con un crecimiento de $0,03 \%$ en comparación con el año anterior, una de las causas como ya se ha mencionado debió ser parte del golpe de la crisis mundial en la economía ecuatoriana, tal como pasó con la desigualdad que tuvo su aumento. En cuanto al año 2015 se observa el pico más alto con un crecimiento de $0,04 \%$, aquí cabe recalcar que la desigualdad también incrementó, mientras que el ingreso per cápita también subió. Esta situación evidencia que el crecimiento no siempre resulta en una disminución de la pobreza.

Tabla 1: Evolución del ingreso per cápita familiar, coeficiente de Gini e incidencia de la pobreza en Ecuador, 2007-2017

\begin{tabular}{lccccccccccc}
\hline Años & $\mathbf{2 0 0 7}$ & $\mathbf{2 0 0 8}$ & $\mathbf{2 0 0 9}$ & $\mathbf{2 0 1 0}$ & $\mathbf{2 0 1 1}$ & $\mathbf{2 0 1 2}$ & $\mathbf{2 0 1 3}$ & $\mathbf{2 0 1 4}$ & $\mathbf{2 0 1 5}$ & $\mathbf{2 0 1 6}$ & $\mathbf{2 0 1 7}$ \\
\hline Ingreso per cápita familiar & 142,35 & 146,38 & 144,01 & 162,32 & 175,13 & 190,88 & 206,39 & 217,10 & 228,71 & 222,81 & 230,17 \\
Coeficiente de Gini & 0,55 & 0,51 & 0,50 & 0,50 & 0,47 & 0,48 & 0,49 & 0,47 & 0,48 & 0,47 & 0,46 \\
Incidencia de la pobreza & 36,74 & 35,09 & 36,03 & 32,76 & 28,64 & 27,31 & 25,55 & 22,49 & 23,28 & 22,92 & 21,46 \\
\hline
\end{tabular}

Fuente: INEC-ENEMDU, 2007-2017.

Elaboración: Autores

Los efectos a nivel nacional se reflejan dentro de cada una de las regiones naturales de Ecuador. A nivel general son las regiones Costa y Sierra la que muestran mejores indicadores. La Sierra posee el ingreso per cápita más alto hasta el 2017, destacándose las provincias de Pichincha y Tungurahua. En cuanto a desigualdad, la región Costa presenta el menor coeficiente de Gini al 2017, con 0.43 . Mientras que, en incidencia de la pobreza, son las dos regiones que rondan entre el $20 \%$ de población considerada pobre. En cuanto a la Amazonía, a pesar de poseer la fuente de mayores ingresos del país (petróleo y minas), es la registra el ingreso per cápita más bajo, la desigualdad y niveles de pobreza más altos. Esto se debe a la alta concentración de capital, mano de obra en las regiones Costa y Sierra, y una falta de políticas redistributivas eficaces, lo que impide que la riqueza generada en la Amazonía pueda beneficiar a su población.

\section{Metodología}

La base de datos utilizada para hacer las siguientes estimaciones del modelo econométrico fue obtenida de la
ENEMDU publicada por el INEC. La muestra que abarca este estudio está compuesta a nivel nacional y por provincias en el periodo comprendido desde 2007 a 2017. Se unifica la provincia de Santo Domingo de los Tsáchilas con Pichincha y la provincia de Santa Elena con Guayas, debido a que se constituyeron como provincias independientes a fines de 2007. También, se une a todas las provincias de la Amazonía como una sola unidad territorial, debido a que antes del año 2014 los datos de la ENEMDU respecto de estas provincias tenían representatividad solo a nivel regional. Por lo tanto, para poder analizar comparativamente todos los años, se considera esta región como una provincia más dentro de la base de datos de panel para todo el periodo 2007-2017. Se excluye Galápagos porque es un territorio independiente al continente y tiene régimen especial, por lo que no se levanta información de esta región a través de la encuesta en mención.

En la estimación, la variable dependiente corresponde a la incidencia de la pobreza por ingreso que está calculada a partir de la proporción de pobres de la población total. Las dos variables explicativas del modelo son el ingreso 
per cápita del hogar y el coeficiente de Gini. La primera, que representa el crecimiento económico, está medida a través del ingreso del hogar dividido para cada uno de los miembros de este y, se determina como el promedio del ingreso per cápita para todas las provincias tomadas para la muestra. Por su parte, la variable de desigualdad está medida con el coeficiente de Gini del ingreso per cápita, que está comprendido 0 y 1 , siendo 1 el indicador de las provincias más desiguales y cero las más equitativas. En este modelo se espera que a medida que crezca el ingreso per cápita la pobreza disminuye, y a medida que disminuya la desigualdad, la pobreza también lo haga.

Por último, el modelo para esta investigación es uniecuacional, donde existe una sola variable dependiente (incidencia de la pobreza) y varias independientes (el ingreso per cápita de las familias y el coeficiente de Gini) con datos de panel de 11 periodos temporales (2007-2017). Además, como el objetivo es demostrar el aporte que tiene el crecimiento económico y la desigualdad en la reducción de la incidencia de la pobreza a manera de elasticidades, y considerando que los datos se ajustan de mejor forma, se ha optado por usar un modelo de regresión logarítmico múltiple, con el que se obtiene el logaritmo natural de cada una de las variables dependiente e independientes para la estimación. Se usa el método clásico de análisis de regresión logarítmica múltiple a través de los Mínimos Cuadrados Ordinarios -MCO- dada la linealidad de los parámetros a estimar, en uso del paquete informático Stata versión 15 para la transformación de las variables y estimaciones de los modelos. Así, el modelo econométrico de los efectos del crecimiento económico y la desigualdad en la incidencia de la pobreza es:

$$
\ln Y=\hat{\beta}_{1}+\hat{\beta}_{2} \ln X_{1}+\hat{\beta}_{3} \ln X_{2}+\hat{\mu}
$$

Donde:

$\ln Y=$ Logaritmo natural de la incidencia de la pobreza. $\ln X_{1}=$ Logaritmo natural del ingreso per cápita familiar. $\ln X_{2}=$ Logaritmo natural del coeficiente de Gini.

$\hat{\beta}_{1}=$ Intercepto o constante del modelo de regresión logarítmico múltiple.

$\hat{\beta}_{2}=$ Elasticidad pobreza-ingreso.

$\hat{\beta}_{3}=$ Elasticidad pobreza-desigualdad.

$\hat{\mu}=$ Término de error estocástico.

\section{Resultados y discusión}

El modelo econométrico indica que, en Ecuador a nivel nacional, la elasticidad del coeficiente de Gini frente a la pobreza es mayor que la elasticidad del ingreso per cápita de las familias
Tabla 2: Modelo econométrico pobreza-ingreso y pobrezadesigualdad a nivel nacional

\begin{tabular}{ll}
\hline \multicolumn{1}{c}{ Variable dependiente } \\
Logaritmo natural de la incidencia de la pobreza \\
\hline Logaritmo natural del ingreso per cápita familiar & $-0,8976^{* * *}$ \\
& $(0,0380)$ \\
Logaritmo natural del coeficiente de Gini & $1.5441^{* * *}$ \\
& $(0,1097)$ \\
Constant & $9,1547^{* * *}$ \\
& $(0,1710)$ \\
Observaciones: 231 & \\
R2: 0,86 & \\
R2 Ajustado: 0,85 & \\
Raíz del error cuadrático medio: 0,15461 & \\
Estadístico $\mathrm{F}: 674,82(\mathrm{df}=2 ; 228)$ & \\
\hline Nota: $* \mathrm{p}<0.1 ; * * \mathrm{p}<0.05 ; * * * \mathrm{p}<0.01$ &
\end{tabular}

Los coeficientes de las variables coinciden con la teoría enunciada en la primera sección. Además, los dos coeficientes de las dos variables independientes son estadísticamente significativas y el modelo en su conjunto también es significativo. La interpretación individual para cada coeficiente de las variables es la siguiente:

$\hat{\beta}_{1}$ (intercepto): Si el ingreso per cápita familiar logarítmico y el coeficiente de Gini logarítmico son 0, la incidencia de la pobreza logarítmica será de $9,55 \%$. Pese a que se ha realizado la interpretación para el intercepto es preciso mencionar que la interpretación de este coeficiente no se debe realizar en el modelo logarítmico, ya que, el logaritmo natural de cero es una indeterminación matemática.

$\hat{\beta}_{2}$ (ingreso per cápita familiar): representa la elasticidad pobreza-ingreso, e indica que, si el ingreso per cápita familiar logarítmico aumenta en el $1 \%$, se espera que la incidencia de la pobreza logarítmica disminuye en promedio un $0,90 \%$.

$\hat{\beta}_{3}$ (coeficiente de Gini): representa la elasticidad pobreza-desigualdad, e indica que, si el coeficiente de Gini logarítmico aumenta en un $1 \%$, se espera que la incidencia de la pobreza logarítmica aumente en 1,54\%.

A través de la estimación del modelo se logró confirmar con la teoría la relación que existe entre el crecimiento económico medido con el ingreso per cápita familiar y la desigualdad medida con el coeficiente de Gini frente a la pobreza. Para el primer caso la relación es negativa, lo que indica que a medida que crece el ingreso per cápita familiar, asumiendo que es un resultado del crecimiento económico, la incidencia de la pobreza disminuye. Para el segundo caso, tomando en cuenta que el coeficiente de Gini va de 0: perfecta igualdad a 1: perfecta desigualdad, la relación es positiva, mientras más crezca el coeficiente de Gini lo que deviene en mayor desigualdad, mayor será la incidencia de la pobreza.

Ambas variables son estadísticamente significativas al $1 \%$ para el modelo y las dos aportan en la disminución de la incidencia de la pobreza. o obstante, como se mide la elasticidad, se confirma también que la elasticidad pobrezadesigualdad es más representativa para la disminución de la incidencia $\mathrm{N}$ de la pobreza a nivel nacional dado que ésta a- 
porta un 0,64\% más (diferencia en valores absolutos de los coeficientes) que el incremento de los ingresos per cápita familiares, elasticidad pobreza-ingreso. Ello ratifica la Hipótesis de Bourguignon, establecida a partir de los resultados obtenidos a través de un modelo econométrico, que explicaba que es necesario una combinación de políticas macroeconómicas de crecimiento y de distribución del ingreso para la disminución de la pobreza.

Los resultados obtenidos en el modelo econométrico coinciden con el estudio de Ravallion \& Chen (1997). En este describe por medio de la elasticidad del crecimiento frente a la pobreza la relación robusta de estas dos variables, con la peculiaridad de tener una mayor incidencia en la pobreza cuando se tiene bajos niveles de desigualdad. Por lo tanto, en cuanto a los resultados presentados se corrobora con el hecho de tener una elasticidad pobreza-ingreso menor a una elasticidad pobreza-desigualdad, que constata un nivel de concentración del ingreso es alta, aunque esté por debajo del nivel de América Latina. Expósito, Fernández \& Velasco (2017) explican en su estudio este fenómeno, el cual encuentra que el crecimiento económico no incide en la pobreza como debería en la región gracias a la concentración de la riqueza.

Andrade, Marinho \& Lima (2017) en su investigación sobre Brasil, a partir de las mismas variables utilizadas en el modelo econométrico realizado, se destacan resultados similares a los obtenidos ya que en los dos casos las políticas de distribución resultan con mayor incidencia de la pobreza. Se tiene en cuenta que los dos países tienen altos niveles de desigualdad. En relación con la investigación de Kalwij \& Vershoor (2004) mencionada, se destaca que los países con amplia población cerca de la línea de la pobreza le resultan más eficaz la combinación de políticas de crecimiento y distribución del ingreso como es el caso de Brasil y Ecuador. Por tal motivo, en Ecuador la elasticidad pobreza-ingreso y la elasticidad pobreza-desigualdad son representativas.

Por su parte, los resultados de las elasticidades obtenidos de las estimaciones mediante las regresiones por provincias y por regiones son:

Tabla 3: Elasticidades a nivel provincial

\begin{tabular}{ccc}
\hline Provincia & Pobreza-Ingreso & Pobreza-Desigualdad \\
\hline Azuay & $-1,1402120$ & 0,7594647 \\
Bolívar & $-0,3402616$ & 1,7085520 \\
Cañar & $-0,8013812$ & 1,1388860 \\
Carchi & $-0,5517023$ & 1,3908560 \\
Cotopaxi & $-0,7844019$ & 1,5493420 \\
Chimborazo & $-0,5575169$ & 0,6205378 \\
El Oro & $-1,1938580$ & 0,6151794 \\
Esmeraldas & $-0,4155495$ & 0,7482608 \\
Guayas & $-0,8903400$ & 1,2072530 \\
Imbabura & $-0,7354283$ & 1,1233930 \\
Loja & $-0,7623280$ & 0,7316675 \\
\hline
\end{tabular}

Tabla 3: Elasticidades a nivel provincial...(continuación)

\begin{tabular}{ccc}
\hline Provincia & Pobreza-Ingreso & Pobreza-Desigualdad \\
\hline Los Ríos & $-0,9230829$ & 0,6127119 \\
Manabí & $-0,9112002$ & 0,9574611 \\
Pichincha & $-1,1569880$ & 1,3708560 \\
Tungurahua & $-0,7738892$ & 1,5159810 \\
Sierra & $-1,0076180$ & 0,9556800 \\
Costa & $-0,8447805$ & 0,9113065 \\
Amazonía & $-0,4152454$ & 0,9506915 \\
\hline
\end{tabular}

Si se observa el cuadro anterior, se denota que la elasticidad pobreza-ingreso es negativa y la elasticidad pobrezadesigualdad es positiva tanto en las provincias como en las regiones ecuatorianas, es decir, que cuando el ingreso per cápita aumenta y se reduce la desigualdad da como resultado la disminución de la pobreza, por lo que se cumple lo mencionado teóricamente. En la Tabla 3 se observa como en 12 de 16 provincias estudiadas la desigualdad tuvo mayor efecto en la incidencia de la pobreza que el ingreso per cápita, esta afirmación corrobora con el estudio realizado por Bourguignon (2004).

En la misma Tabla 3, se observa los resultados por regiones naturales ecuatorianas, la Costa y la Amazonía presentan las elasticidades pobreza-desigualdad con un mayor efecto sobre la incidencia de la pobreza, mientras que en la Sierra la elasticidad pobreza-ingreso tiene un mayor efecto sobre la incidencia de la pobreza. Además, cabe recalcar que la elasticidad pobreza-ingreso y la elasticidad pobrezadesigualdad son representativas en las tres regiones. La única excepción es la Amazonía en cuanto a la elasticidad pobreza-ingreso, ya que, esta es baja con respecto a las demás elasticidades. Por lo tanto, en la Amazonía el ingreso incide en menor medida en la incidencia de la pobreza.

En las regresiones econométricas por provincias y por regiones se ha obtenido que los dos tipos de elasticidades son representativas para la disminución en la incidencia de la pobreza. Para la elasticidad pobreza-ingreso es negativa, lo que quiere decir que ante un aumento del ingreso per cápita de los hogares se da una disminución en la incidencia de la pobreza. La elasticidad pobreza-desigualdad es positiva, lo que quiere decir que ante un aumento de la desigualdad se obtendría un aumento en la incidencia de la pobreza. En las provincias de Azuay, Loja, El Oro, Los Ríos y Orellana las políticas de crecimiento económico resultarían en una disminución en la incidencia de la pobreza, mientras que, en las demás las políticas de disminución de la desigualdad resultarían en una disminución en la incidencia de la pobreza.

Las elasticidades por región dieron como resultado que la región Sierra tenga una mayor significancia en la disminución de la incidencia de la pobreza a través de los ingresos per cápita con una pequeña diferencia de 0,05 de la desigualdad. Además, se toma en cuenta que los ingresos per cápita medios de la región Sierra son los más altos a nivel nacional, por lo cual en la Sierra servirían políticas 
de las dos clases. Por su lado, la región Costa presenta que las políticas de desigualdad surtirían más efecto que las de crecimiento, ya que, se observa una elasticidad pobrezadesigualdad de 0,07 mayor que la elasticidad pobrezaingreso, aquí cabe recalcar que la región Costa es la menos desigual de Ecuador. Por otro lado, en la Amazonía la región más desigual y con menor ingreso per cápita, la disminución de la desigualdad influencia con mayor efecto en la incidencia de la pobreza en comparación al aumento del ingreso per cápita.

Los resultados obtenidos de estos modelos por provincias y por regiones confirman la hipótesis teórica de Bourguignon (2004) donde afirma que las dos políticas en su conjunto son efectivas en la disminución de la incidencia de la pobreza. Por otro lado, también coincide con la investigación de Andrade, Marinho \& Lima (2017) realizada en Brasil y analizada por Estados, en la cual, la elasticidad pobreza-ingreso es menor en los lugares donde el ingreso medio es bajo, esto ocurre en la Amazonía donde existen efectos menos profundos sobre la pobreza a partir del ingreso per cápita y son más profundos los cambios en la reducción de la pobreza a partir de políticas encaminadas a la disminución del nivel de desigualdad, por el contrario donde existe mayor desigualdad es donde incide más las políticas de desigualdad sobre la disminución en la incidencia de la pobreza que las políticas de crecimiento, como es el caso de la Sierra y Amazonía.

A modo de síntesis, estos modelos permiten analizar lo que se debería aplicar en cuanto a política pública que este encaminada a la disminución de la incidencia de la pobreza, de la misma forma, permite resaltar la realidad de cada provincia y región en cuanto a elegir la política que resulte más beneficiosa para disminuir la incidencia de la pobreza ya sea esta de crecimiento o de disminución de la desigualdad.

Dada la confirmación de la hipótesis planteada, con base en la revisión del Plan de Desarrollo Nacional de Ecuador -PND- 2013-2017 (que recoge la información desde el 2007) es necesario contrastar las metas con los resultados del periodo y los retos que el país tendría frente al triángulo crecimiento, pobreza y desigualdad.

De acuerdo con el PND 2013 - 2017, en el 2007 la pobreza por ingreso a nivel nacional fue del $36,7 \%$, en el 2012 fue del $27,3 \%$ y la meta propuesta fue del $20 \%$. Según datos de la ENEMDU en el 2017 la tasa de pobreza se ubicó en $21,5 \%$. Con respecto a la pobreza para los años 2007 y 2012, se registró 16,9\% y 11,2\%, respectivamente; y, se propuso como meta llegar al $3 \%$. Sin embargo, de acuerdo los datos del 2017 de la ENEMDU, Ecuador registró una tasa del 7,9\%

Con relación a la desigualdad, en el 2007, Ecuador registró un coeficiente de Gini del 0,55, en el 2012 se registró 0,47 y el PND 2013-2017 estableció llegar al 0,44. Conforme a los datos de la ENEMDU del 2017 el país registró un coeficiente del 0,46 . Por otro lado, la brecha entre el $10 \%$ más rico y el $10 \%$ más pobre en 2012 fue de
41,7 veces, en el 2012 fue de 26,1 veces y el PND estableció para el 2017 llegar a 20 veces; sin embargo en el 2017 la brecha fue de 24,3 veces.

Es importante mencionar que el promedio de crecimiento del PIB real de Ecuador entre el 1996 y el 2006 fue del 3,1\%, en tanto que entre los años 2007-2017 fue del $3,3 \%$ (Banco Central del Ecuador, 2020). Adicionalmente, se debe anotar que el crecimiento del ingreso familiar fue del $62 \%$ entre el periodo 2007-017. Uno de los retos fundamentales es al menos mantener tasas de crecimiento económico superior al crecimiento poblacional que entre el 2010 y 2020 se estimó tasa promedio de 1,6\% (Sistema Nacional de Información, 2017). Lamentablemente de no tener una economía dinámica y resiliente, los logros alcanzados podrían verse afectados.

A la luz de los resultados, pese a que Ecuador no cumplió con las metas propuestas por el PND del 2017, los resultados de la reducción de la pobreza son positivos, el crecimiento del periodo 2007 - 2017 tuvo un mejor desempeño económico que el del 1996-2006; y los resultados en cuanto a la reducción de la desigual también fueron significativos. En este sentido vemos que ambas variables han contribuido al decremento de la pobreza; no obstante, gracias al modelo econométrico se encontró que la vinculadas a la distribución tuvieron un mayor efecto sobre la reducción de la pobreza.

\section{Conclusiones y recomendaciones}

La revisión el estado del arte lleva a concluir que la aplicación diferenciada de políticas de crecimiento o desigualdad para erradicar la pobreza depende del contexto de la zona de implementación. No obstante, en concordancia con la Hipótesis Bourguignon, se expone que ambas políticas son necesarias, el solo centrarse en una de ellas podría ocasionar, en el caso de solo crecimiento económico, mayor concentración del ingreso y, en el caso de distribución, falta de ingresos.

En Ecuador, según datos de la ENEMDU 2007-2017, los indicadores de pobreza y desigualdad tienen una tendencia decreciente. Además, el ingreso per cápita de los hogares va en aumento. A nivel regional, la Sierra y la Costa presentan los niveles más bajos de pobreza y desigualdad. No obstante, la Amazonía, a pesar de los abundantes recursos que posee, presenta alta incidencia de la pobreza, la mayor desigualdad y en promedio el menor ingreso per cápita.

A partir de los resultados obtenidos en el modelo econométrico nacional se concluye que a pesar de que las dos elasticidades pobreza-ingreso y pobreza-desigualdad reducen la incidencia de la pobreza, la elasticidad pobrezadesigualdad incide con mayor fuerza en la incidencia de la pobreza que la elasticidad pobreza-ingreso. Además, en los modelos por provincias y regiones se concluye que la elasticidad que reduce en mayor proporción la incidencia de la pobreza depende de la situación de cada provincia o región. Si la provincia o región presenta altos niveles 
de desigualdad, su elasticidad pobreza-desigualdad es más alta que la elasticidad pobreza-ingreso. Por otro lado, si la provincia o región presenta bajos niveles de ingreso su elasticidad pobreza-ingreso es mayor que la elasticidad pobreza desigualdad. Así, para el caso de Ecuador, dados sus niveles de desigualdad, requiere de importantes políticas de distribución y redistribución.

Ecuador es un país con alta concentración del ingreso, y considerando los problemas productivos estructurales que atañen al país, en épocas de bonanza es necesario el óptimo suo de los recursos. He ahí la importancia de tener preparadas estrategias en políticas públicas o propuestas que, analizando el contexto real del país, sugieran alternativas que dinamicen más los beneficios generados del crecimiento. Se recomienda que las políticas distributivas y redistributivas se enfoquen justamente en las zonas, provincias y en la población que, por su condición, no gozan del mismo éxito en cuanto a calidad de vida, que los demás. El aplicar las políticas diferenciadas podría llevar a beneficiar en mayor proporción a la población en situación de pobreza y pobreza extrema.

Se recomienda que los actores que lideran las políticas públicas tomen en cuenta que las políticas encaminadas a la reducción de la desigualdad son más efectivas para reducir la incidencia de la pobreza en comparación con las políticas de crecimiento para el caso de Ecuador. No obstante, no se puede dejar de lado la aplicación de políticas que promuevan el crecimiento económico, debido que cualquier política no puede llevarse a cabo sin recursos y una economía dinámica y resiliente. Consideramos que al momento de tratar las políticas de distribución y redistribución se privilegie mecanismos tributarios directos y progresivos como plantea la Constitución de Ecuador del 2008 en su artículo número 300.

\section{Referencias Bibliográficas}

Acemoglu, D., Johnson, S., y Robinson, J. (2005). The rise of europe: Atlantic trade institutional change and economic growth. American Economic Review.

Andrade, J., Marinho, E., y Lima, G. (2017). Crecimiento económico y concentración del ingreso: sus efectos en la pobreza del brasil. Descargado de https://repositorio.cepal.org/ bitstream/handle/11362/42693/1/ RVE123_Araujo.pdf

Banco Mundial. (1990). Descargado de http://econ -worldbank .org/external/default/ main?pagePK=64165259\&theSitePK= $469372 \& \mathrm{pi}-\mathrm{PK}=64165421 \&$ menuPK= $64166093 \&$ entityID $=000425962$ _20130228141712

Bourguignon, F. (2004). The poberty-growthinequality triangle. Descargado de http:// siteresources. worldbank.org/INTPGI/ Resources/3426741206111890151/ 15185_ICRIER_paper-final.pdf
Bruno, M., Ravallion, M., y Squire, L. (1996). Equidad y el crecimiento en los paises en desarrollo: viejas y nuevas perspectivas sobre los temas de política. Washington D.C: Banco Mundial.

CEPAL. (2017). Comision economica para america latina y el caribe. Descargado de https://repositorio.cepal.org/ bitstream/handle/11362/42716/7/ S1800002_es.pdf

Chen, S., y Wang, Y. (2001). China's growth and poverty reduction: recent trends between 1990 and 1999 (Inf. Téc.). World Bank.

Expósito, A., Fernández, J., y Velasco, F. (2017). Crecimiento economico, pobreza y desigualdad. Revista de economía mundial, 47.

Fondo Monetario Internacional. (2014). Informe anual 2014 del fondo monetario internacional. Washintong D.C.: FMI

INEC. (s.f.). Ecuador en cifras. Descargado de http://www. ecuadorencifras.gob.ec/ documentos/web-inec/POBREZA/2017/ Diciembre/Reporte (Obtenido de Pobreza y Desigualdad)

INEC. (2018). Encuesta nacional de empleo, desempleo y subempleo: Indicadores de pobreza y desigualdad. Descargado de http://www.ecuadorencifras.gob.ec/ documentos/web-inec/POBREZA/2018/ Diciembre-2018/201812_Pobreza.pdf (Obtenido de Instituto Nacional de Estadísticas y Censos)

Kalwij, A., y Verschoor, A. (2004). How good is growth for the poor? the role of the initial income distribution in regional diversity in poverty trends. Descargado de https://papers.ssrn.com/sol3/ papers.cfm?abstract_id=639503 (Obtenido de SSRN ELibrary Statistics:)

Kuznets, S. (1955). Economic growth and income inequality. Nueva York: American Economic Review.

López, J. D. (2017). Rankia méxico. Descargado de https://www.rankia.mx/blog/ abc-de-economia-y-finanzas/

3534526-hipotesis-kuznets (Obtenido de El ABC de la economía y fiananzas: Hipotesis de Kuznets)

Naciones Unidas. (2015). Objetivos y metas de desarrollo sostenible. Descargado de https://www. un - org/sustainabledevelopment/es/ objetivos-de-desarrollo-sostenible/ (Objetivos de Desarrollo Sostenible)

Oxfam. (2019). Las causas de la pobreza en el mundo. ingredientes que suman. Descargado de https://blog.oxfamintermon.org/las -causas-de-la-pobreza-en-el-mundo/

Piketty, T. (2013). El capital del siglo xxi. Paris: Harvard University Press. 
PNUD. (2019). Programa de las naciones unidas para el desarrollo. obtenido de objetivo 1: Fin de la pobreza. Descargado de http:// WWw . undp.org/content/undp/es/home/ sustainable-development-goals/ goal-1-no-poverty.html

Ravallion, M., y Chen, S. (1997). What can new survey data tell us about recent changes in distribution and poverty? (Vol. 11). Washington DC.: Banco Mundial.

Revista Gestión. (2013). ¿cómo se originó la peor crisis financiera de la historia? Descargado de https : / / gestion.pe/economia/origino-peor -crisis-financiera-historia-48101

Secretaría Nacional de Planificación y Desarrollo, Senplades. (2017, septiembre). Plan nacional de desarrollo 2017 - 2021. Descargado de https://www.planificacion.gob.ec/ wp-content/uploads / downloads/2017/ 10 /PNBV-26-OCT-FINAL_OK.compressed1 .pdf

Sistema Nacional de Información. (2017). Proyecciones $y$ estudios demográficos - sistema nacional de información. estimaciones y proyecciones de población. Descargado de https:// sni.gob.ec/proyecciones-y-estudios - demograficos

Stewart, F. (2000). Distribuição de renda e desenvolvimento. Brasilia: NEAD.

Stiglitz, J. (2015). La gran brecha. Madrid: Taurus.

Verdera, F. (2005). Biblioteca virtual clacso. Descargado de http://bibliotecavirtual .clacso.org.ar/ar/libros/coedicion/ verdera/03desigua.pdf (Obtenido de Desigualdad y Pobreza en el Perú)

Recibido: 26 de junio de 2020

Aceptado: 24 de marzo de 2020

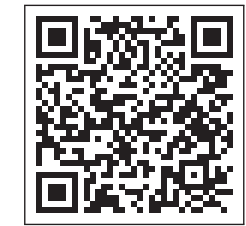

